\title{
Nervio Occipital Mayor: Trayecto, Relaciones Anatómicas e Implicancias Clínicas de sus Posibles Sitios de Atrapamiento
}

\author{
Great Occipital Nerve: Course, Anatomical Relations and \\ Clinical Implications of their Potential Entrapment Sites
}

\author{
Castillo-Rebolledo, Daniela ${ }^{1}$; Riveros, Andrés ${ }^{2,3}$; Sousa-Rodrigues, Celio Fernando ${ }^{4}$ \& Olave, Enrique ${ }^{5}$
}

CASTILLO-REBOLLEDO, D. ; RIVEROS, A.; SOUSA-RODRIGUES, C. F. \& OLAVE, E. Nervio occipital mayor: Trayecto, relaciones anatómicas e implicancias clínicas de sus posibles sitios de atrapamiento. Int. J. Morphol., 38(5):1235-1243, 2020.

RESUMEN: El nervio occipital mayor (NOM) se forma del ramo dorsal del nervio espinal C2 y asciende entre la musculatura cervical posterior para inervar la piel del cuero cabelludo. Diversos autores han descrito su recorrido, sin embargo, es escasa la información referente a la relación que presenta este nervio con el músculo oblicuo inferior de la cabeza (OIC) y su trayecto intramuscular. El objetivo de este estudio fue determinar el recorrido y relaciones que el NOM estableció en el intervalo existente entre los músculos OIC y músculo trapecio (T). Para ello, se midieron las distancias verticales y horizontales a la altura de la protuberancia occipital externa y línea mediana, y se dividió al músculo OIC en tercios para observar variaciones del recorrido de este nervio. Junto con medir el diámetro del NOM, se midieron las distancias vertical y horizontal de este nervio a través de cinco puntos de referencia muscular y un punto de referencia vascular. Estos puntos musculares fueron: a) sobre el vientre del músculo OIC (punto 1); b) en la cara profunda del músculo semiespinoso de la cabeza (SEC) (punto 2); c) en la cara superficial del músculo SEC (punto 3); d) en la cara profunda del músculo T (punto 4); y e) en la cara superficial del músculo T (punto 5). A este se sumó el punto 6, en el cual se establecieron las distancias vertical y horizontal con la arteria occipital a la altura de la cara superficial del músculo T. Para ello se disecaron 18 cabezas (36 triángulos suboccipitales) de cadáveres adultos brasileños pertenecientes al laboratorio de Anatomía de la Universidade Federal de Alagoas (UFAL), Maceió, Brasil. Las distancias verticales y horizontales obtenidas respecto de los seis puntos fueron: 63,67 y 27,15 mm (punto 1); 53,89 y 21,44 mm (punto 2); 30,61 y 14,49 mm (punto 3); 20,39 y 22,8 mm (punto 4); 5,86 y 33,46 mm (punto 5); 5,99 y 35,56 mm (punto 6), respectivamente. En relación al músculo OIC, el NOM se ubicó en un 72,22 \% de las muestras en el tercio medio de este músculo, 19,44 $\%$ en su tercio lateral y un $8,33 \%$ en su tercio medial. Todos estos hallazgos deben ser considerados al momento de diagnosticar correctamente posibles atrapamientos del NOM en la región cervical profunda, siendo además, una contribución para el éxito de procedimientos quirúrgicos de esta región.

PALABRAS CLAVE: Anatomía; Inervación; Nervio occipital mayor; Músculo oblicuo inferior de la cabeza; Triángulo suboccipital; Variaciones anatómicas.

\section{INTRODUCCIÓN}

El nervio occipital mayor (NOM) se origina del ramo medial del ramo dorsal del nervio espinal $\mathrm{C} 2$, aunque también puede recibir fibras derivadas del ramo dorsal del nervio espinal C3 (Gawel \& Rothbart, 1992). Emerge posteriormente entre la primera y la segunda vértebra cervical, y luego se curva para ascender alrededor del margen inferior del músculo oblicuo inferior de la cabeza (OIC) y así recorrer la cara dorsal del músculo recto posterior mayor de la cabeza (Schaeffer, 1953; Testut \& Latarjet, 1972; Bogduk,
1982; Mosser et al., 2004; Rouvière \& Delmas, 2005; Standring et al., 2008). A continuación, este nervio atraviesa al músculo semiespinoso de la cabeza (SEC) y luego inmediatamente inferior a la línea nucal superior perfora al músculo trapecio (T). Finalmente cruza a través del tendón del músculo T junto con la arteria occipital y continúa su recorrido hasta el vértice del cráneo en donde recoge la sensibilidad general del tegumento del cuero cabelludo. En este último tramo, el NOM establece comunicaciones hacia

\footnotetext{
${ }^{1}$ Programa de Magíster en Ciencias Morfológicas, Universidad de La Frontera, Temuco, Chile.

${ }^{2}$ Departamento de Ciencias Morfológicas, Facultad de Medicina y Ciencia, Universidad San Sebastián, Lientur 1457, Concepción 4080871, Chile.

${ }^{3}$ Programa de Doctorado en Ciencias Morfológicas, Universidad de La Frontera, Temuco, Chile.

${ }^{4}$ Universidade Federal de Alagoas, Maceió, Brasil.

${ }^{5}$ Facultad de Medicina, Universidad de La Frontera, Temuco, Chile.
} 
medial con el nervio occipital tercero y hacia lateral con el nervio occipital menor (Hollinshead, 1968; Gawel \& Rothbart; Standring). El trayecto antes descrito tiene un curso tortuoso lo que lo hace susceptible a la compresión y a presentar múltiples puntos de atrapamiento que desencadenan una posible neuralgia occipital (Lazorthes \& Gaubert, 1956; Mosser et al.; Ducic et al., 2009; Janis et al., 2010; Cesmebasi et al., 2015).

Respecto de las lesiones del NOM, Natsis et al. (2006) describieron posibles causas para el desarrollo de la neuralgia occipital. Entre ellas destacó la irritación del NOM en su origen producto de artritis en el segmento C1-C2 (Hunter \& Mayfield, 1949; Stechison \& Mullin, 1994), o el atrapamiento de este nervio cuando perfora al músculo SEC o el tendón del músculo T. También consideraron que el atrapamiento se puede presentar en la región suboccipital, en específico en su relación con el músculo OIC (Vital et al., 1989). Scherer et al. (2019) señalaron que éste músculo puede desempeñar un papel importante en el síndrome del dolor occipital ya que durante la flexión del cuello aumenta el espacio atlantooccipital y atlantoaxial causando una elongación del NOM en sentido vertical. Lo contrario ocurriría durante el movimiento de extensión, permitiendo la distensión del nervio.

En relación a la búsqueda de estudios que hayan propuesto puntos topográficos en los intervalos musculares de la región cervical tanto profunda como superficial, y que realizaron mediciones respecto a las relaciones anatómicas del NOM en diferentes sitios de atrapamiento miofascial, Janis et al., informaron que el punto de atrapamiento más profundo se ubica dentro de la fascia del vientre del músculo OIC, cercano al proceso espinoso de la segunda vértebra cervical, situándose en promedio a 77,38 mm inferior a la protuberancia occipital externa (POE) y a 20,13 mm desde la línea mediana. Asimismo, Scherer et al. disecaron 40 NOM y lo ubicaron a $68 \mathrm{~mm}$ inferior a la POE y 35,6 mm lateral a la línea mediana, aportando hallazgos significativos respecto a la relación del NOM con el músculo OIC, ya que midieron la interacción que presentó este nervio alrededor del músculo (a), su incorporación en la fascia densa superficial (b), o su relación con una manga miofascial dentro del músculo; midiendo además la longitud y grosor del músculo OIC.

Por otra parte, Natsis et al. registraron las distancias cuando el NOM perforó al músculo SEC, alcanzando 27,2 mm y $12,1 \mathrm{~mm}$, vertical desde la POE y horizontal desde línea mediana, respectivamente. De la misma forma, Vital et al. registraron estas mediciones a $37,3 \mathrm{~mm}$ vertical a la POE y $11,5 \mathrm{~mm}$ horizontal a la línea mediana. Lo anterior se replicó en las experiencias de Mosser et al., quienes concluyeron que a nivel de la cara superficial del músculo SEC, el NOM emerge a $28,9 \mathrm{~mm}$ inferior a la POE y $13,95 \mathrm{~mm}$ lateral a la línea mediana, resultados que para Ducic et al., alcanzaron 30,2 $\mathrm{mm}$ inferior a la POE y 14,9 $\mathrm{mm}$ lateral a la línea mediana. Por su parte, Janis et al. registraron estas mismas mediciones, sin embargo, las establecieron tanto cuando el NOM perforó la cara profunda de músculo SEC como también cuando este nervio realizó su emergencia en la cara superficial del mismo músculo. Sus distancias vertical y horizontal en la cara profunda del músculo SEC alcanzaron $59,71 \mathrm{~mm}$ y $17,46 \mathrm{~mm}$, versus $10 \mathrm{~s} 34,52 \mathrm{~mm}$ y 15,52 $\mathrm{mm}$, que se registraron en las mediciones vertical y horizontal realizadas en la cara superficial del mismo músculo.

Respecto a la relación del NOM con el músculo T, Vital et al., informaron que este nervio se dividió en ramos subcutáneos terminales antes o después de atravesar la estrecha abertura que le ofreció este músculo. En este sentido, Natsis et al. determinaron que al medir la distancia vertical desde la POE hasta el punto en que el NOM atravesó el músculo $\mathrm{T}$, existió una diferencia significativa entre cadáveres masculinos (promedio de 11,4 $\mathrm{mm}$, rango 8,1-14 mm, DE 1,7 ) y femeninos (promedio de 7,9 mm, rango 3,9-11,3 mm, DE 1,9). En esta misma línea, si bien Janis et al. realizaron las mismas mediciones, reportaron diferencias notorias en las distancias que el NOM presentó en las caras superficial y profunda del músculo T. Producto de ello, le otorgó al grosor del músculo una importancia topográfica a la hora de establecer las correctas relaciones entre el NOM y el músculo T.

Cabe agregar que Janis et al., además de medir puntos topográficos de relación miofascial, reportaron las mediciones existentes entre el NOM y la arteria occipital en la región nucal. Sus registros alcanzaron un promedio de 10,67 $\mathrm{mm}$ inferior a la POE y $30,27 \mathrm{~mm}$ lateral a la línea mediana.

Si bien los estudios citados proporcionaron puntos topográficos de referencia para la identificación del NOM; existen importantes diferencias si estas mediciones son realizadas en la cara profunda o superficial de los músculos SEC y músculo T. Lo anterior refleja que existe un desacuerdo entre los autores con respecto a los puntos de referencias idóneos para determinar el correcto trayecto del NOM en la región cervical profunda y superficial, a lo que se suma la carencia de estos reportes en nuestra zona geográfica.

Basado en lo anterior, el objetivo del presente estudio fue determinar el recorrido y las principales relaciones que el NOM estableció en el intervalo entre el músculo OIC y el músculo T. Lo anterior no sólo contribuirá al conocimiento anatómico específico de esta zona de interés clínico, sino que permitirá al profesional de la salud tener una guía al momento de realizar procedimientos de uso clínico evitando producir lesiones que afecten al NOM. 


\section{MATERIAL Y MÉTODO}

Se utilizaron 18 cadáveres (36 regiones triángulos suboccipitales), de las cuales 32 pertenecieron a muestras masculinas y cuatro a muestras femeninas de individuos adultos brasileños, pertenecientes al laboratorio de Anatomía de la Universidade Federal de Alagoas (UFAL), Maceió, Brasil. Estos cadáveres se encontraban fijados bajo inmersión en una solución con formaldehído al $10 \%$.

Esta investigación se realizó utilizando una metodología de carácter cuantitativo, no experimental, transeccional y descriptiva, para lo cual se procedió a realizar una detallada disección de la región cervical posterior, utilizando para ello material quirúrgico "ad-hoc".

Previo a la realización de la disección, los cadáveres se ubicaron en posición decúbito prono y se afeitaron sus cabezas, para luego ser marcadas con un rotulador indeleble realizando una línea vertical desde la POE hasta el proceso espinoso de la séptima vértebra cervical y una línea horizontal ubicada entre los márgenes inferiores de los procesos mastoídeos del hueso temporal (línea intermastoídea).

El inicio de esta disección requirió la remoción de la piel y el tejido subcutáneo, dejando así expuesta la línea nucal superior, el músculo esternocleidomastoídeo (ECM) y los músculos T. Una vez localizado el NOM, la disección se enfocó en seguir el recorrido de este nervio desde superficial a profundo a través de la musculatura de esta zona. Para realizar las mediciones con las estructuras relacionadas con el trayecto de este nervio, se encontró y se aisló a la arteria occipital. Posteriormente se identificó el músculo T, liberando su fascia en las caras superficial y cara profunda. En seguida se prescindió de las fibras del ECM, así mismo, se cortó y traccionaron las fibras superiores del músculo $\mathrm{T}$, dejando expuesto al músculo esplenio de la cabeza. Este último músculo fue removido para alcanzar el músculo SEC, cuyas caras superficial y profunda fueron cuidadosamente disecadas; de esta forma pudo ser identificado el ingreso del NOM a los planos cervicales profundos. Utilizando la técnica de divulsión se accedió al triángulo suboccipital, y en este punto se individualizaron los músculos que conforman este triángulo, con especial interés en el músculo OIC, requieriendo para esto eliminar el panículo adiposo y el plexo venoso de esta zona.

Del trayecto del NOM en esta zona, se registraron 6 puntos topográficos en los cuales este nervio estableció una relación anatómica de interés, ya sea por su estrecha cercanía o por el hecho de que el trayecto incluyó atravesar un vientre muscular. En concreto los puntos en donde se realizaron mediciones fueron:

a) Punto 1: Sobre el vientre del músculo OIC.

b) Punto 2: En la cara profunda del músculo SEC.

c) Punto 3: En la cara superficial del músculo SEC.

d) Punto 4: En la cara profunda del músculo T.

e) Punto 5: En la cara superficial del músculo T.

f) Punto 6: Relación con la arteria occipital.

Para establecer mediciones objetivas del trayecto de este nervio, en cada uno de los puntos considerados se establecieron dos distancias: a) una distancia vertical, la cual se obtuvo trazando una línea perpendicular a la altura de la POE, y b) una distancia horizontal que se obtuvo desde la línea mediana (Fig. 1). Dentro de las medidas antropométricas de cabeza y cuello, también se midió la longitud desde la POE al proceso espinoso de la segunda vértebra cervical; y la longitud intermastoídea, la cual se estableció entre los márgenes inferiores de los procesos mastoídeos.

Respecto del punto 1, además de medir las distancias ya mencionadas con el músculo OIC, se midió la longitud del músculo y se dividió su vientre en tercios. De esta forma se pudo establecer en qué tercio el NOM se relacionó con este músculo. Adicionalmente en los puntos 1 al 4 se incluyó el registro del diámetro (D) del NOM (D1 para el punto 1, D2 para el punto 2, D3 para el punto 3 y D4 para el punto 4 ).

Todos los datos fueron ingresados a una base de datos Microsoft Excel ${ }^{\circledR}$ y se calcularon las medidas de tendencia central de cada una de las variables registradas. Por último, se registraron las variantes anatómicas.

Todas las mediciones fueron realizadas con un caliper digital Mitutoyo ${ }^{\circledR}$ de $0,01 \mathrm{~mm}$ de precisión y cada una de las muestras disecadas fueron fotografiadas con una cámara réflex digital Nikon ${ }^{\circledR}$ D5300 y se realizó un esquema que consideró el trayecto y distribución del NOM.

\section{RESULTADOS}

El NOM se encontró en la totalidad de las muestras. Cada uno de los puntos (1 al 6) fueron encontrados y medidos. Los resultados se resumen en la Tabla I, junto a sus promedios, desviaciones estándar y rangos mínimo/ máximo. 


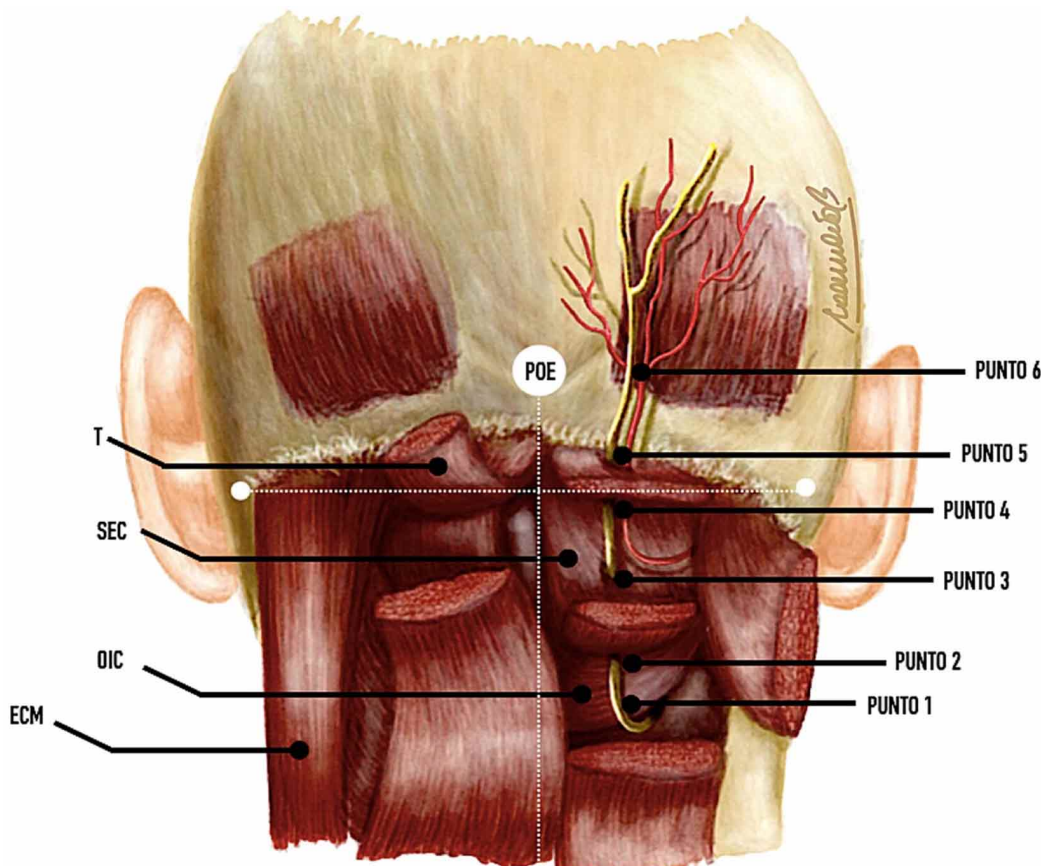

Fig 1. Trayecto del nervio occipital mayor (NOM) en la región cervical y sus puntos de compresión miofascial, junto a su relación vascular con la arteria occipital. POE: Protuberancia occipital externa, T: Músculo trapecio, SEC: Músculo semiespinoso de la cabeza, OIC: Músculo oblicuo inferior de la cabeza, ECM: Músculo esternocleidomastoídeo. Punto 1: Sobre el vientre muscular de OIC; Punto 2: Perforando la cara profunda del músculo SEC; Punto 3: Emergiendo por la cara superficial del músculo SEC, Punto 4: Perforando la cara profunda del músculo T; Punto 5: Emergiendo por la cara superficial del músculo T; Punto 6: Relación vascular con la arteria occipital.

El punto 1 consideró la relación del NOM con el vientre del músculo OIC, el cual se ubicó a una distancia vertical promedio de $63,67 \mathrm{~mm}$ y a una distancia horizontal promedio de $27,15 \mathrm{~mm}$.

El punto 2 y punto 3 consideraron la relación del NOM con la cara profunda y superficial del músculo SEC, respectivamente. El punto 2 se ubicó a una distancia vertical promedio de $53,89 \mathrm{~mm}$ y a una distancia horizontal promedio de 21,44 mm; mientras que el punto 3 se registró a una distancia vertical promedio de $30,61 \mathrm{~mm}$ y a una distancia horizontal promedio de $14,49 \mathrm{~mm}$.
Así mismo, el punto 4 y punto 5 consideraron la relación del NOM con la cara profunda y superficial del músculo $\mathrm{T}$, respectivamente. El punto 4 se localizó a una distancia vertical promedio de 20,39 $\mathrm{mm}$ y a una distancia horizontal promedio de $22,8 \mathrm{~mm}$; mientras que el punto 5 se situó a una distancia vertical promedio de $5,86 \mathrm{~mm}$ y a una distancia horizontal promedio de $33,46 \mathrm{~mm}$.

Respecto a la única relación vascular considerada, el punto 6 midió la relación del NOM con la arteria occipital, la cual se dispuso a una distancia vertical promedio de $5,99 \mathrm{~mm}$ y a una distancia horizontal promedio de $35,56 \mathrm{~mm}$, ubicándose lateral al NOM en la totalidad de las muestras.

Las diferencias de lateralidad de las distancias vertical y horizontal se resumen en la Tabla II, no encontrando diferencias significativas.

En relación a las medidas antropométricas de cabeza y cuello consideradas, éstas arrojaron un valor promedio de $67,87 \pm 6,97 \mathrm{~mm}$ (rango de 54,83 $\mathrm{mm}-$ $81,68 \mathrm{~mm}$ ) para la distancia entre la POE y el proceso espinoso de la segunda vértebra cervical; y de 123,51 $\pm 8,47 \mathrm{~mm}$ (rango de $112,91 \mathrm{~mm}-137,66 \mathrm{~mm}$ ) para la distancia intermastoídea.

Complementando las mediciones del punto 1 , además se incluyó la longitud del músculo OIC la cual registró un valor promedio de 50,17 $\pm 4 \mathrm{~mm}$ (rango de 43,59 a 57,25 $\mathrm{mm}$ ). Del total de las muestras, el 72,22 \% de los casos se ubicó sobre el tercio medio del músculo, el 19,44\% se situó sobre el tercio lateral y un $8,33 \%$ se ubicó sobre el tercio medial (Fig. 2).

Tabla I. Distancia vertical y horizontal de los puntos considerados en el trayecto del NOM, considerando la totalidad de la muestra.

\begin{tabular}{|c|c|c|c|c|c|c|c|c|}
\hline & \multicolumn{4}{|c|}{ Distancia vertical } & \multicolumn{4}{|c|}{ Distancia horizontal } \\
\hline & Promedio & D.E & Mín. & Máx. & Promedio & D.E & Mín. & Máx. \\
\hline & $(\mathrm{mm})$ & $(\mathrm{mm})$ & $(\mathrm{mm})$ & $(\mathrm{mm})$ & $(\mathrm{mm})$ & $(\mathrm{mm})$ & $(\mathrm{mm})$ & $(\mathrm{mm})$ \\
\hline Punto 1 & 63,67 & 6,81 & 50,23 & 83,67 & 27,15 & 3,12 & 21,74 & 34,96 \\
\hline Punto 2 & 53,89 & 4,18 & 45,37 & 63,54 & 21,44 & 3,61 & 14,93 & 29,33 \\
\hline Punto 3 & 30,61 & 5,84 & 22,2 & 42,02 & 14,49 & 3,57 & 8,92 & 21,39 \\
\hline Punto 4 & 20,39 & 3,73 & 14,21 & 29,04 & 22,8 & 3,83 & 15,18 & 30,37 \\
\hline Punto 5 & 5,86 & 1,32 & 3,5 & 8,38 & 33,46 & 4,03 & 24,5 & 44,9 \\
\hline Punto 6 & 5,99 & 1,38 & 4,4 & 10,51 & 35,56 & 3,99 & 26,89 & 49,67 \\
\hline
\end{tabular}

*Mín.: Mínimo / Máx.: Máximo 
Respecto a los diámetros (D) del NOM, el promedio de este nervio obtuvo un valor de $4,65 \mathrm{~mm}$ para D1 (en el punto 1); 3,67 mm para D2 (en el punto 2); $3,65 \mathrm{~mm}$ para D3 (en el punto 3 ) y finalmente $4,61 \mathrm{~mm}$ para D4 (en el punto 4). Los resultados de todos los diámetros considerados se resumen en la Tabla III junto a sus promedios, desviaciones estándar y rangos mínimo/ máximo.

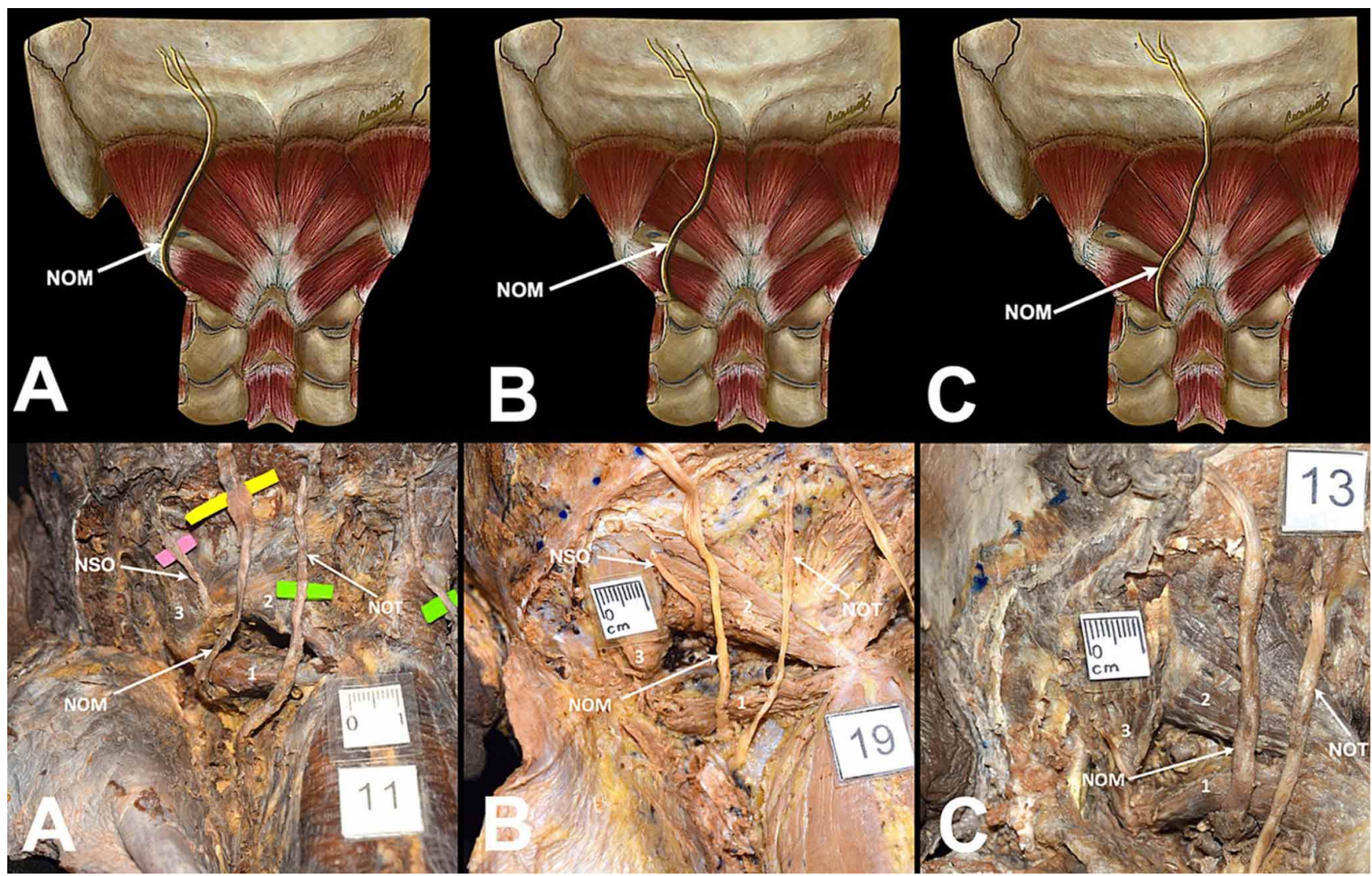

Fig 2. Variaciones del recorrido del nervio occipital mayor (NOM) sobre el músculo oblicuo inferior de la cabeza (OIC) en el triángulo suboccipital. A) NOM ubicado sobre el tercio lateral del músculo OIC, B) NOM ubicado sobre el tercio medio del músculo OIC, C) NOM ubicado sobre el tercio medial del músculo OIC. NSO: Nervio suboccipital, NOT: Nervio occipital tercero, 1) músculo OIC, 2) músculo recto posterior mayor de la cabeza, 3) músculo oblicuo superior de la cabeza.

Finalmente y en relación a las variantes anatómicas, en siete casos $(19,44$ $\%$ ) el NOM se dividió en ramos terminales previamente a su ingreso por la cara profunda del músculo $\mathrm{T}$, en donde cuatro muestras correspondieron al lado derecho y tres al lado izquierdo, cuyos ramos presentaron una longitud promedio de 4,29 $\pm 0,9$ (rango de 3,43 mm $5,78 \mathrm{~mm}$ ). Por otra parte, sólo en una de las muestras del lado izquierdo $(2,77 \%)$ se observó que el NOM se dividió en dos ramos terminales inmediatamente después de emerger a través de la cara superficial del SEC. Estos ramos presentaron una longitud promedio de 27,89 $\mathrm{mm}$ para el ramo medial y de $29,53 \mathrm{~mm}$ para el ramo lateral (Fig. 3).

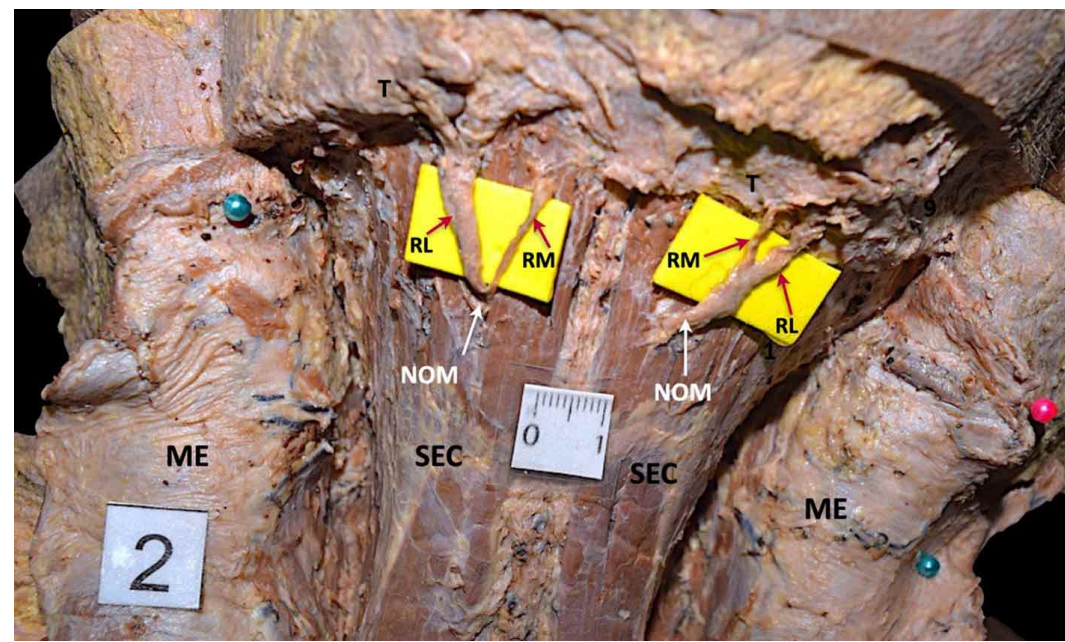

Fig 3. Región cervical posterior a nivel de la cara superficial del músculo semiespinoso de la cabeza (SEC). T: músculo trapecio, ME: músculo esplenio de la cabeza, NOM: Nervio occipital mayor, RL: ramo lateral, RM: ramo medial. 


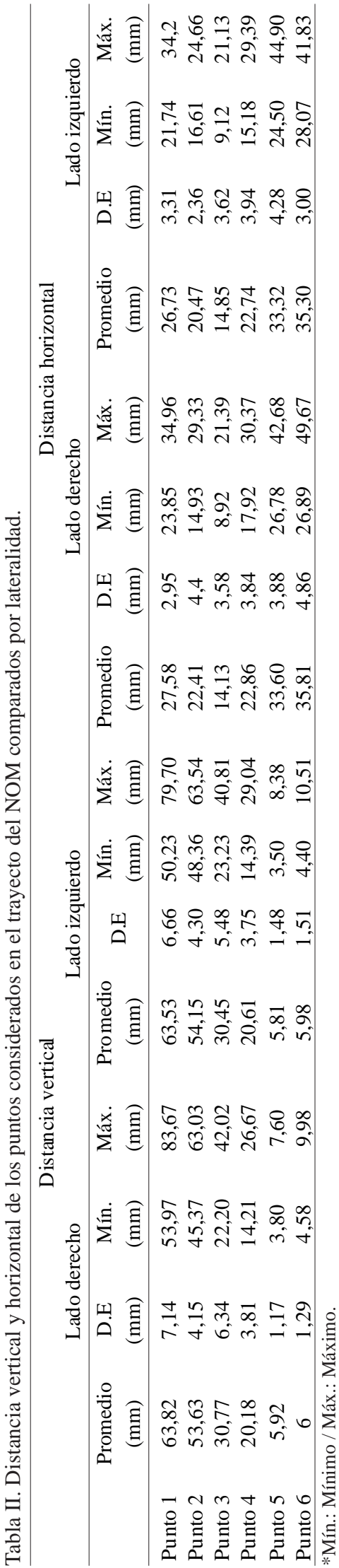

Tabla III. Diámetro del NOM en los puntos 1 al 4 durante su trayecto.

\begin{tabular}{|c|c|c|c|c|}
\hline \multicolumn{5}{|c|}{ Diámetro NOM } \\
\hline & $\begin{array}{c}\text { Promedio } \\
(\mathrm{mm})\end{array}$ & $\begin{array}{c}\text { D.E } \\
(\mathrm{mm})\end{array}$ & $\begin{array}{l}\text { Mín. } \\
\text { (mm) }\end{array}$ & $\begin{array}{l}\text { Máx. } \\
(\mathrm{mm})\end{array}$ \\
\hline D1 (Punto 1) & 4,65 & 0,73 & 2,6 & 5,92 \\
\hline D2 (Punto 2) & 3,67 & 0,84 & 1,94 & 5,52 \\
\hline D3 (Punto 3) & 3,65 & 0,9 & 2 & 5,19 \\
\hline D4 (Punto 4) & 4,61 & 0,98 & 3,1 & 6,81 \\
\hline
\end{tabular}

*Mín.: Mínimo / Máx.: Máximo.

\section{DISCUSIÓN}

El NOM ha correspondido a un nervio ampliamente descrito en la literatura y en investigaciones previas respecto a su recorrido y a sus relaciones anatómicas, sin embargo aún existen desacuerdos en base a su ubicación topográfica y diámetros, considerando además la carencia de información que existe en la relación de este nervio con el músculo OIC.

Respecto a las mediciones que realizaron otros autores en relación a nuestro punto 1, Janis et al. disecaron 50 NOM y registraron a este nervio a nivel del vientre del músculo OIC a un promedio de 77,38 $\mathrm{mm}$ inferior a la POE y a 20,13 $\mathrm{mm}$ lateral a la línea mediana. Replicando lo anterior, Scherer et al. informaron medidas similares y describieron la ubicación del NOM a una distancia vertical y horizontal de $68 \mathrm{~mm}$ y $35,6 \mathrm{~mm}$, respectivamente. Ambos resultados discrepan con nuestros hallazgos, ya que para las mismas distancias obtuvimos una promedio de $63,67 \mathrm{~mm}$ para la distancia vertical y $27,15 \mathrm{~mm}$ para la distancia horizontal. Estos valores pueden ser variables sobre todo si se tiene en cuenta que no se habían considerado en investigaciones previas las relaciones del NOM con los tercios del músculo OIC para observar las variaciones del recorrido de este nervio.

Considerando a continuación el punto 2, Janis et al. describieron al NOM penetrando la cara profunda del músculo SEC a $59,71 \mathrm{~mm}$ para la distancia vertical y 17,46 mm para distancia horizontal; mientras que para Israel et al. (2018), la distancia vertical fue de $52 \mathrm{~mm}$ (rango de $40-67 \mathrm{~mm}$, DE $8 \mathrm{~mm}$ ) y la distancia horizontal fue de $14 \mathrm{~mm}$ (rango, 3 - $32 \mathrm{~mm}$, DE $6 \mathrm{~mm}$ ). En comparación con nuestros hallazgos, los resultados fueron variables, ya que para la distancia vertical inferior a la POE obtuvimos un promedio de $53,89 \mathrm{~mm}$ y $21,44 \mathrm{~mm}$ para la distacia horizontal respecto a la línea mediana. Éstas diferencias podrían estar relacionadas con la concentración del panículo adiposo situado en el interior del triángulo suboccipital, el cual podría desplazar el trayecto del NOM antes de penetrar la cara profunda del músculo SEC. Nuestra experiencia en la disección de esta zona describe que las concentraciones de tejido adiposo dentro y alrededor del triángulo suboccipital son muy variables entre el sexo masculino y femenino, sumando además las características fisonómicas de cada individuo.

En relación al punto 3, sitio en donde el NOM emerge a través de la cara superficial del músculo SEC, son diversas las experiencias documentadas en trabajos previos. Tal es el caso de Mosser et al., quienes disecaron 40 NOM obteniendo medidas de 28,9 $\mathrm{mm}$ para la distancia vertical y $13,95 \mathrm{~mm}$ para la distancia horizontal. Estos resultados fueron similares a los obtenidos por Natsis et al., quienes disecaron 40 cadáveres (29 mujeres y 11 hombres) e identificaron los puntos donde el NOM emerge del músculo SEC y músculo $\mathrm{T}$, detectando una diferencia derecha-izquierda a nivel del músculo $\mathrm{T}$ pero no 
en el músculo SEC. El NOM atravesó al músculo SEC a una distancia de 27,2 $\mathrm{mm}$ (rango de 15,8 - 38,8 $\mathrm{mm}$, DE 6,9 mm) inferior a la POE, y de $12,1 \mathrm{~mm}$ (rango de 6,2-18,7 mm, DE 2,4 mm) lateral a la línea mediana, mediciones que fueron similares en hombres y mujeres. Experiencias similares fueron las realizadas por Vital et al., y Janis et al., ya que Vital et al., registraron mediciones para las distancias vertical y horizontal de $37,7 \mathrm{~mm}$ y $11,5 \mathrm{~mm}$; mientras que para Janis et al. los valores obtenidos fueron de $34,52 \mathrm{~mm}$ y $15,52 \mathrm{~mm}$, respectivamente. A lo anterior se suma la experiencia de Israel et $a l$. , quienes informaron para las mismas distancias un valor de $36 \mathrm{~mm}$ para la distancia vertical y $16 \mathrm{~mm}$ para la distancia horizontal, en una muestra intraoperatoria de $60 \mathrm{NOM}$ en donde la mayoría correspondía a individuos de sexo femenino ( $88 \%$ ). Nuestros resultados son más coincidentes a lo obtenido por Ducic et al., quienes publicaron una serie anatómica de 250 NOM de los cuales 112 correspondieron a muestras intraoperatorias y 13 a cadáveres formolizados. Sus hallazgos registraron al NOM a 30,2 mm inferior a la POE y $14,9 \mathrm{~mm}$ lateral a la línea mediana; resultados que para nosotros fueron de $30,61 \mathrm{~mm}$ y 14,49 en las distancias vertical y horizontal. Tales diferencias entre las medidas obtenidas por los autores y nuestro trabajo se apoyan en lo documentado por Ducic et al., ya que observaron que el NOM tenía un curso intramuscular a través del músculo SEC en el 98,5\% de los casos y que era asimétrico en la cara superficial de éste músculo en el $44 \%$ de los pacientes, concluyendo que la variabilidad observada entre los lados derecho e izquierdo y entre individuos, tiene implicaciones directas en la aplicación de bloqueos nerviosos y descompresión quirúrgica.

Respecto a las distancias obtenidas en el punto 4, en donde el NOM perfora la cara profunda del músculo T, nuestros hallazgos registaron al NOM a 20,39 $\mathrm{mm}$ inferior a la POE para la distancia vertical y a $22,80 \mathrm{~mm}$ lateral a la línea mediana para la distancia horizontal. Estos resultados fueron muy similares a los obtenidos por Janis et al., quienes en el mismo punto registraron una distancia vertical de $21 \mathrm{~mm}$ y $24 \mathrm{~mm}$, respectivamente. En relación a este punto, no encontramos investigaciones significativas que aportaran más información sobre las mediciones de este sitio anatómico.

En relación al punto 5, sitio en que el NOM emerge por la cara superficial del músculo T, Natsis, et al. informaron al NOM a $8,9 \mathrm{~mm}$ en la distancia vertical y $34,65 \mathrm{~mm}$ en la distancia horizontal. Éstos resultados fueron similares aunque ligeramente elevados para Vital et al., quienes para las mismas distancias obtuvieron valores de $11,5 \mathrm{~mm}$ y 37,3 $\mathrm{mm}$, respectivamente. Nuestros hallazgos se acercan más a los de Janis et al., ya que obtuvieron para las distancias vertical y horizontal valores de $4,36 \mathrm{~mm}$ y $37,07 \mathrm{~mm}$; valores que en nuestra investigación fueron de $5,86 \mathrm{~mm}$ y 33,46 $\mathrm{mm}$, respectivamente.
La única relación vascular que se incluyó en nuestro trabajo fue la proximidad con la arteria occipital a la cual situamos en el punto 6. Esta arteria se ubicó superficialmente, profundamente o rodeando al NOM, pero siempre distribuyéndose lateral a este nervio (100\% de la muestra). El único autor que aporta datos significativos respecto a las mismas medidas consideradas en nuestra investigación es Janis et al., quienes informaron que esta relación se ubica de forma variable, a veces en la profundidad del músculo $\mathrm{T}$ caudal a la línea horizontal a través de la POE, o bien, superolateral a esta zona más cerca del oído. Sus medidas obtuvieron valores de $10,67 \mathrm{~mm}$ en la distancia vertical y $30,27 \mathrm{~mm}$ en la distancia horizontal; resultados no tan coincidentes con los nuestros, ya que para las mismas distancias registramos a la arteria occipital a 5,99 $\mathrm{mm}$ inferior a la POE en la distancia vertical, y a 35,56 mm lateral a la línea mediana en la distancia horizontal. Estos datos indudablemente son un aporte, ya que no habían sido considerados en investigaciones similares.

En la tabla IV se presenta un resumen comparativo entre los datos aportados por los autores previamente.

Respecto a las relaciones que el NOM estableció en los tercios del músculo OIC a nivel del punto 1, el 72,22 \% de las muestras se situó sobre el tercio medio, un 19,44\% se ubicó sobre su tercio lateral y un $8,33 \%$ se registró sobre su tercio medial. Creemos que estos hallazgos adoptan significativa importancia frente a un diagnóstico de neuralgia occipital. Este punto topográfico a nivel del vientre del músculo OIC podría utilizarse como sitio específico para realizar las descompresiones quirúrgicas proximales del NOM cuando otros puntos topográficos del trayecto de este nervio no logren ser identificados debido a su tortuoso y variable recorrido a través de la región cervical posterior.

En relación a las mediciones del diámetro (D) del NOM, se estableció realizar mediciones sólo en los puntos del 1 al 4 (desde el vientre del músculo OIC hasta la cara profunda del músculo T), ya que en el 80,55 \% de las muestras presentó un tronco nervioso que atravesó por el músculo T para luego formar ramos terminales desde su cara superficial; razón por la cual sólo consideramos las mediciones del tronco nervioso previo a su división. Los valores de los diámetros de cada uno de los puntos considerados (D1, D2, D3 y D4) muestran un ensanchamiento del NOM hacia su curso periférico específicamente entre el músculo SEC y el músculo $\mathrm{T}$, exceptuando la relación intramuscular con el músculo SEC, ya que inicialmente su promedio fue de 4,65 $\mathrm{mm}$ al ubicarse sobre el músculo OIC, pero luego se redujo al relacionarse con la cara profunda y superficial del músculo SEC, obteniendo medidas de $3,67 \mathrm{~mm}$ y $3,65 \mathrm{~mm}$, respectivamente. Esto demuestra la influencia que tiene éste 


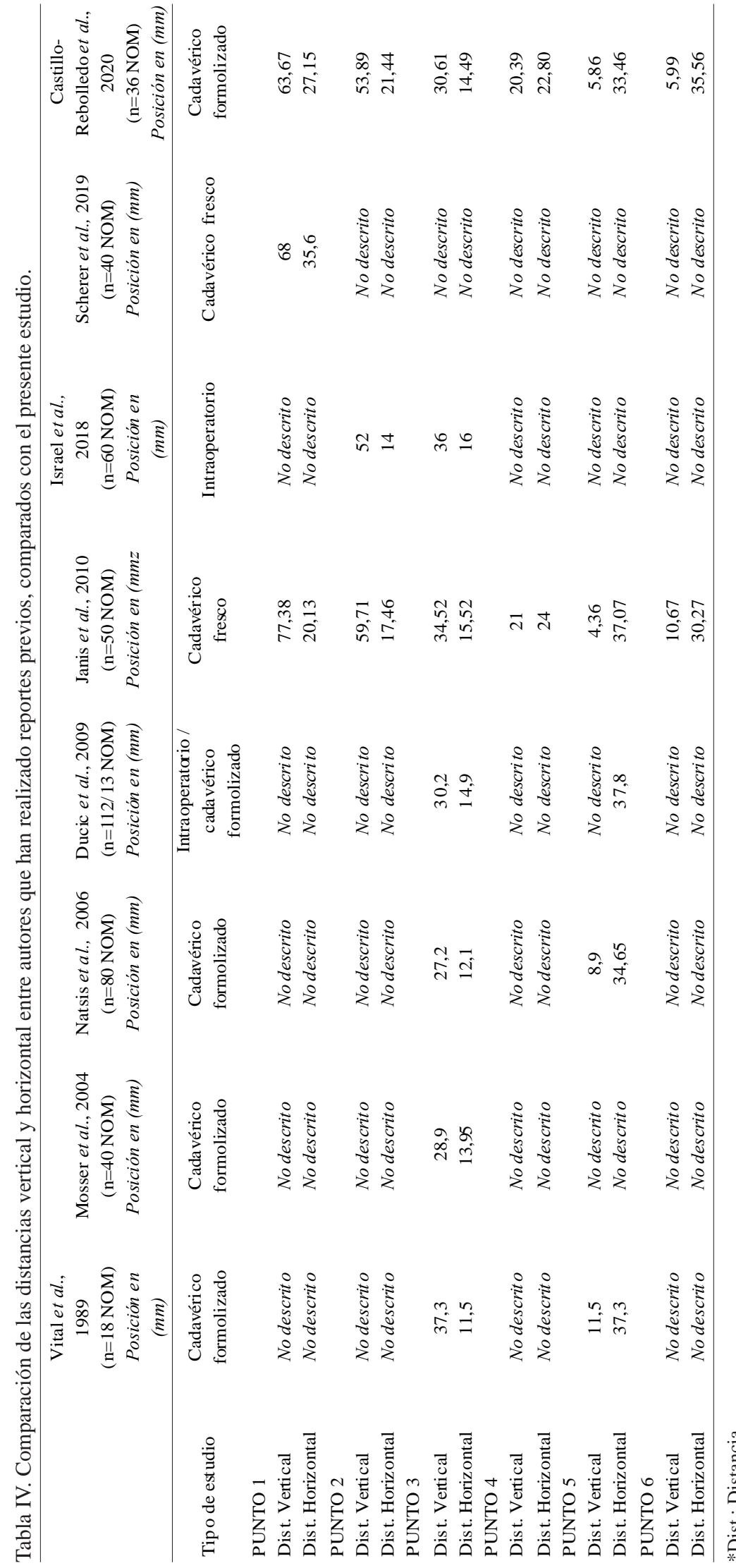

músculo sobre el recorrido del NOM durante el trayecto intramuscular. Finalmente a nivel de la cara profunda del músculo T, el nervio presentó un diámetro promedio de 4,61 mm. A estos resultados se les puede comparar con lo reportado por Natsis et al., quienes registraron valores por debajo de los nuestros para D3 con un ancho de 2,35 mm; mientras que Mosser et al., para el mismo punto informaron un valor de $2,7 \mathrm{~mm}$.

Finalmente, concluimos que este estudio tiene varios hallazgos significativos. En primer lugar, la relación que el NOM tiene con el músculo OIC, especialmente considerando su ubicación y diámetro, ya que según nuestras mediciones las coordenadas lo sitúan a 63,67 $\mathrm{mm}$ por debajo de una línea trazada a la altura de la POE, y a $27,15 \mathrm{~mm}$ lateral a la línea mediana, presentándose en un $72,22 \%$ en el tercio medio de este músculo con un diámetro promedio de $4,65 \mathrm{~mm}$.

En segundo lugar, creemos que el punto en que el NOM se relaciona con el músculo OIC (punto 1) debe considerarse al diagnosticar y realizar un tratamiento de neuralgia occipital cuando la identificación de éste nervio no ha tenido éxito en sitios más superficiales. Como se demostró, hay muchas investigaciones previas que basan sus estudios en el recorrido del NOM especialmente a nivel del músculo SEC, sin embargo y hasta ahora, no se había otorgado mayor interés clínico a la relación que existe entre el NOM y el músculo OIC en la región cervical profunda. Con los antecedentes antes informados creemos que se debe prestar atención específica a su descompresión proximal a nivel de su interacción con el músculo OIC, ya que podría constituir un sitio óptimo para su tratamiento.

Por último, creemos que todos los hallazgos obtenidos servirán de guía a los anatomistas, investigadores y profesionales de la salud que requieran más información sobre el curso periférico variable de este nervio, ya que clínicamente, un amplio conocimiento de su trayecto resultará de utilidad para el tratamiento de los síndromes de la neuralgia occipital. 
AGRADECIMIENTOS: Se agradece al ilustrador Sr. Christian Caamaño Binimelis por sus contribuciones gráficas a esta comunicación científica.

CASTILLO-REBOLLEDO, D. ; RIVEROS, A.; SOUSARODRIGUES, C. F. \& OLAVE, E. Great occipital nerve: Course, anatomical relations and clinical implications of their potential entrapment sites. Int. J. Morphol., 38(5):1235-1243, 2020.

SUMMARY: The great occipital nerve (GON) is formed from the dorsal branch of the $\mathrm{C} 2$ spinal nerve and ascends between the posterior cervical musculature to innervate the skin of the scalp. Various authors have described its course, however, there is little information regarding the relationship that this nerve presents with the obliquus capitis inferior (OCI) and its intramuscular path. The objective of this study was to determine the route and relationships that the GON established in the interval between the OCI muscles and the trapezius muscle (T). For this, the vertical and horizontal distances were measured at the height of the external occipital protuberance and median line, and the OCI muscle was divided into thirds to observe variations in the path of this nerve. Along with measuring the diameter of the GON, the vertical and horizontal distances of this nerve were measured through five muscle reference points and one vascular reference point. These muscle points were: a) on the belly of the OCI muscle (point 1); b) in the deep face of the semispinalis capitis muscle (SCM) (point 2); c) on the surface of the SCM (point 3); d) on the deep face of the T (point 4); and e) on the surface face of the $\mathrm{T}$ (point 5). To this was added point 6 , in which the vertical and horizontal distances were established with the occipital artery at the height of the superficial face of the T. For this, 18 heads (36 suboccipital triangles) of Brazilian adult corpses belonging to the Anatomy laboratory of the Universidade Federal de Alagoas (UFAL), Maceió, Brazil, were dissected. The vertical and horizontal distances obtained with respect to the six points were: 63.67 and $27.15 \mathrm{~mm}$ (point 1); 53.89 and $21.44 \mathrm{~mm}$ (point 2); 30.61 and $14.49 \mathrm{~mm}$ (point 3); 20.39 and $22.8 \mathrm{~mm}$ (point 4); 5.86 and $33.46 \mathrm{~mm}$ (point 5); 5.99 and 35.56 $\mathrm{mm}$ (point 6), respectively. In relation to the OCI, the GON was located in $72.22 \%$ of the samples in the middle third of this muscle, $19.44 \%$ in its lateral third and $8.33 \%$ in its medial third. All these findings should be considered when correctly diagnosing possible entrapments of GON in the deep cervical region, being a contribution to the success of surgical procedures in this region.

KEY WORDS: Anatomy; Innervation; Great Occipital Nerve; Obliquus Capitis Inferior; Suboccipital triangle; Anatomical Variations.

\section{REFERENCIAS BIBLIOGRÁFICAS:}

Bogduk, N. The clinical anatomy of the cervical dorsal rami. Spine (Phila Pa 1976), 7(4):319-30, 1982

Cesmebasi, A.; Muhleman, M. A.; Hulsberg, P.; Gielecki, J.; Matusz, P.; Tubbs, R. S. \& Loukas, M. Occipital neuralgia: anatomic considerations. Clin. Anat., 28(1):101-8, 2015.
Ducic, I.; Moriarty, M. \& Al-Attar, A. Anatomical variations of the occipital nerves: implications for the treatment of chronic headaches. Plast. Reconstr. Surg., 123(3):859-63, 2009.

Gawel, M. J. \& Rothbart, P. J. Occipital nerve block in the management of headache and cervical pain. Cephalalgia, 12(1):9-13, 1992.

Hollinshead, W. Anatomy for Surgeons. $2^{\mathrm{a}}$ ed. New York, Harper \& Row, 1968.

Hunter, C. R. \& Mayfield, F. H. Role of the upper cervical roots in the production of pain in the head. Am. J. Surg., 78(5):743-51, 1949.

Israel, J. S.; Kempton, S. J. \& Afifi, A. M. Prospective analysis of the greater occipital nerve location in patients undergoing occipital nerve decompression. Ann. Plast. Surg., 81(1):71-4, 2018.

Janis, J. E.; Hatef, D. A.; Ducic, I.; Reece, E. M.; Hamawy, A. H.; Becker, S. \& Guyuron, B. The anatomy of the greater occipital nerve: Part II. Compression point topography. Plast. Reconstr. Surg., 126(5):156372, 2010.

Lazorthes, G. \& Gaubert, J. L'innervation des articulations interapophysaire vertebrales. Compt. Rend. Assoc. Anat., 43:488-94, 1956.

Mosser, S. W.; Guyuron, B.; Janis, J. E. \& Rohrich, R. J. The anatomy of the greater occipital nerve: implications for the etiology of migraine headaches. Plast. Reconstr. Surg., 113(2):693-7, 2004.

Natsis, K.; Baraliakos, X.; Appell, H. J.; Tsikaras, P.; Gigis, I. \& Koebke, J. The course of the greater occipital nerve in the suboccipital region: a proposal for setting landmarks for local anesthesia in patients with occipital neuralgia. Clin. Anat., 19(4):332-6, 2006.

Rouvière, H. \& Delmas, A. Anatomía Humana. Descriptiva, Topográfica y Funcional. 11 a ed. Barcelona, Masson, 2005.

Schaeffer, J. P. Morris's Human Anatomy. A Complete Systematic Treatise. $11^{a}$ ed. New York, McGraw Hill, 1953.

Scherer, S. S.; Schiraldi, L.; Sapino, G.; Cambiaso-Daniel, J.; Gualdi, A.; Peled, Z. M.; Hagan, R. \& Pietramaggiori, G. The greater occipital nerve and obliquus capitis inferior muscle: anatomical interactions and implications for occipital pain syndromes. Plast. Reconstr. Surg., 144(3):730-6, 2019.

Standring, S. Gray's Anatomy. The Anatomical Basis of Clinical Practice. $40^{\mathrm{a}}$ ed. Edinburgh, Churchill Livingstone/Elsevier, 2008.

Stechison, M. T. \& Mullin, B. B. Surgical treatment of greater occipital neuralgia: an appraisal of strategies. Acta Neurochir. (Wien), 131(34):236-40, 1994.

Testut, L. \& Latarjet, A. Tratado de Anatomía Humana. $9^{\mathrm{a}}$ ed. Barcelona, Salvat, 1972.

Vital, J. M.; Grenier, F.; Dautheribes, M.; Baspeyre, H.; Lavignolle, B. \& Sénégas, J. An anatomic and dynamic study of the greater occipital nerve (n. of Arnold). Applications to the treatment of Arnold's neuralgia. Surg. Radiol. Anat., 11(3):205-10, 1989.

\author{
Dirección para correspondencia: \\ Dr. Enrique Olave \\ Facultad de Medicina \\ Universidad de La Frontera \\ Casilla 54-D \\ Temuco \\ CHILE
}

Email: enrique.olave@ufrontera.cl

Recibido : 10-03-2020

Aceptado: 16-04-2020 